\title{
Multimodal image fusion via deep generative models
}

Giovanna Maria Dimitri $(1,5)^{*}$, Simeon Spasov (1)*, Andrea Duggento (2), Luca Passamonti $(3,6)$, Pietro Lio'(1), Nicola Toschi $(2,4)$

1) University of Cambridge, Cambridge, Department of Computer Science and Technology, William Gates Building, 15 JJ Thomson Ave, Cambridge, CB3 0FD, UK

2) Department of Biomedicine and Prevention, University of Rome "Tor Vergata", Via Montpellier 1, 00133, Roma, RM, Italy

3) Department of Clinical Neurosciences, University of Cambridge, Herschel Smith Building, Forvie Site, Robinson Way, Cambridge Biomedical Campus, Cambridge, CB2 0SZ, Cambridge, UK

4) A.A. Martinos Center for Biomedical Imaging, Massachusetts General Hospital and Harvard Medical School, Boston, USA

5) Dipartimento di Ingegneria dell'Informazione e Scienze Matematiche (DIISM), University of Siena, Italy

6) Istituto di Bioimmagini e Fisiologia Molecolare (IBFM), Consiglio Nazionale delle Ricerche (CNR) Via F.1li Cervi, 93, 20090, Segrate, Milano, Italy.

*The two authors contributed equally to this work, gmd43@cl.cam.ac.uk 


\begin{abstract}
Recently, it has become progressively more evident that classic diagnostic labels are unable to accurately and reliably describe the complexity and variability of several clinical phenotypes. This is particularly true for a broad range of neuropsychiatric illnesses such as depression and anxiety disorders or behavioural phenotypes such as aggression and antisocial personality. Patient heterogeneity can be better described and conceptualized by grouping individuals into novel categories, which are based on empirically-derived sections of intersecting continua that span both across and beyond traditional categorical borders. In this context, neuroimaging data carry a wealth of spatiotemporally resolved information about each patient's brain. However, they are usually heavily collapsed a priori through procedures which are not learned as part of model training, and consequently not optimized for the downstream prediction task. This is due to the fact that every individual participant usually comes with multiple whole-brain 3D imaging modalities often accompanied by a deep genotypic and phenotypic characterization, hence posing formidable computational challenges.

In this paper we design and validate a deep learning architecture based on generative models rooted in a modular approach and separable convolutional blocks (which result in a 20 -fold decrease in parameter utilization) in order to a) fuse multiple 3D neuroimaging modalities on a voxel-wise level, b) efficiently convert them into informative latent embeddings through heavy dimensionality reduction, c) maintain excellent generalizability and minimal information loss. As proof of concept, we test our architecture on the well characterized Human Connectome Project database ( $\mathrm{n}=974$ healthy subjects), demonstrating that our latent embeddings can be clustered into easily separable subject strata which, in turn, map to extremely different phenotypical information (including organic, neuropsychological, personality variables) which was not included in the embedding creation process.

The ability to extract meaningful and separable phenotypic information from brain images alone can aid in creating multi-dimensional biomarkers able to chart spatio-temporal trajectories which may correspond to different pathophysiological mechanisms unidentifiable to traditional data analysis approaches. In turn, this may be of aid in predicting disease evolution as well as drug response, hence supporting mechanistic disease understanding and also empowering clinical trials.
\end{abstract}

\title{
1. Introduction
}

Over the last years, it is has become progressively more evident that classic diagnostic labels are unable to accurately and reliably describe the complexity and variability of several clinical phenotypes. This is particularly true for a broad range of neuropsychiatric illnesses such as depression and anxiety disorders or behavioural phenotypes such as aggression and antisocial personality. In neurology and psychiatry, the problem is compounded by the clear lack of boundaries between normal and abnormal behaviour and by the presence of often overlapping diagnostic categories. The need for a transdiagnostic psychiatry and for a brain-based categorization of the clinical entities that transcend the traditional Diagnostic Standard Manual (DSM) labels has been emphasized several times. Alzheimer's Disease (AD) and Parkinson's Disease (PD) are paradigmatic examples. Both diseases manifest themselves with a myriad of symptoms and signs which may or may not occur across different patients, and typically can be linked to extremely heterogeneous timescales and trajectories (i.e. evolutions over time). Accordingly, post-mortem studies have confirmed an extremely high heterogeneity in neuropathological findings not only in AD (Rabinovici et al., 2017) but also in other, classically unrelated, but most probably somehow dimensionally connected neurological syndromes (Adler et al, 2010).

To overcome this issue, trans-diagnostic approaches that rely on a more nuanced and mechanistic representation of the demographic and clinical features of each individual have been proposed. More specifically, patient heterogeneity can be better described and conceptualized by grouping individuals into novel categories, which are based on empirically-derived sections of intersecting continua that span both across and beyond traditional categorical borders. Such novel patient groupings are likely to be 
disjoint and possibly become independent from the currently employed disease categories. This is because a single patient condition often transcends the traditional taxonomies. In contrast, it is reasonable to visualize and describe each patient as occupying their own, unique position in a highdimensional space that depends on specific pathophysiological mechanisms. The uniqueness of this position in such a high-dimensional space is likely to translate into new opportunities to optimize the diagnosis and treatment to the individual needs of each patient ('personalized medicine'). However, the possibility to translate this into clinical practice can only depend on the possibility to access the multidimensional biomarker space that defines the uniqueness of each patient's neurocognitive and behavioural "profile".

This conceptual paradigm is often termed precision- and (in some cases) personalized medicine, which lies in stark contrast with the more commonly employed "one size fits all" approach. The latter is still pervasive in most clinical disciplines, although exceptions exist like e.g. in oncology, where some degree of personalization has been clinically successful. Such observations highlight the urgent need for more objective classification criteria and frameworks, which need to be based on measurable and reproducible biomarkers. This can be achieved by employing deep generative models that are able to meaningfully group individuals (healthy or with clinical disorders) on the basis of key brain structural measures.

Neuroimaging data carry a wealth of spatiotemporally resolved information about each patient's brain. However, they are usually heavily collapsed a priori through procedures which are not learned as part of model training, and consequently not optimized for the downstream prediction task. Still, for a comprehensive data-driven stratification, all relevant pathophysiological mechanisms should be wellrepresented in the multidimensional data fed into the aggregation and pattern recognition frameworks. In this context, the recent appearance of several large multicentre and multimodal, curated large (between 1000 and 40000 individuals at the time of writing) data repositories (e.g. the Parkinson Progression Marker Initiative (PPMI) (Jennings et al., 2011), the Alzheimer Disease Neuroimaging Initiative (ADNI) (Mueller et al., 2005), the UK Biobank initiative (Sudlow et al., 2015), the Cam-CAN dataset (Taylor et al 2017) and the Human Connectome Project (HCP) (Van Essen et al., 2013) is providing novel formidable opportunities as well as challenges. On one hand, this amount of information which has never previously been accessible to neuroimaging researchers: every individual participant usually comes with multiple whole-brain imaging modalities of $10^{5}-10^{6}$ voxels each, often acquired at multiple timepoints. Importantly, these data are usually accompanied by a deep geno/phenotypic characterization (e.g. genetic, biochemical, bio humoral, and neuropsychological markers). This opens up avenues to robust cross-modality data fusion, and therefore to subsequent reduction into embeddings that are fine-grained enough to 1) inform mechanistic hypotheses about disease physiology as well as 2) about the neural substrates determining currently unexplainable withindisease variability. On the other hand, the computational and conceptual challenges in designing data reduction architectures able to exploit voxel-wise multimodal 3D imaging data (most deep learning frameworks are designed to learn from 2D images) while retaining realistic computation times and, crucially, extract informative embeddings while reducing data dimensionality by at least a factor 1000 , are severe.

The aim of this paper is therefore to design and validate a deep learning (DL) architecture based on generative models rooted in a modular approach and separable convolutional blocks. Our goal is to efficiently extract low level informative embeddings while A) fusing multiple 3D neuroimaging modalities on a voxel-wise level B) performing heavy dimensionality reduction with minimal information loss and C) building an architecture able to efficiently reconstruct brain images. This latter aspect is in fact gaining much attention in the field of DL and neuroimaging, mainly with the development of Generative Adversarial Networks aimed to obtain images reconstruction (Yang et al 2020)

As proof of concept, we test our architecture on the well characterized Human Connectome Project (HCP) dataset, where we used multiple modalities to perform our experiments such as JAC, NDI, FA and T1-W brain scans. We demonstrate that our latent multimodal embeddings can be clustered into easily separable subject strata. Additionally, these strata map to extremely different phenotypical information (including organic, neuropsychological, personality variables) which was not included in the embedding creation process. 


\section{Materials and Methods}

A comprehensive overview of the workflow adopted in this paper is presented in Fig. 1.

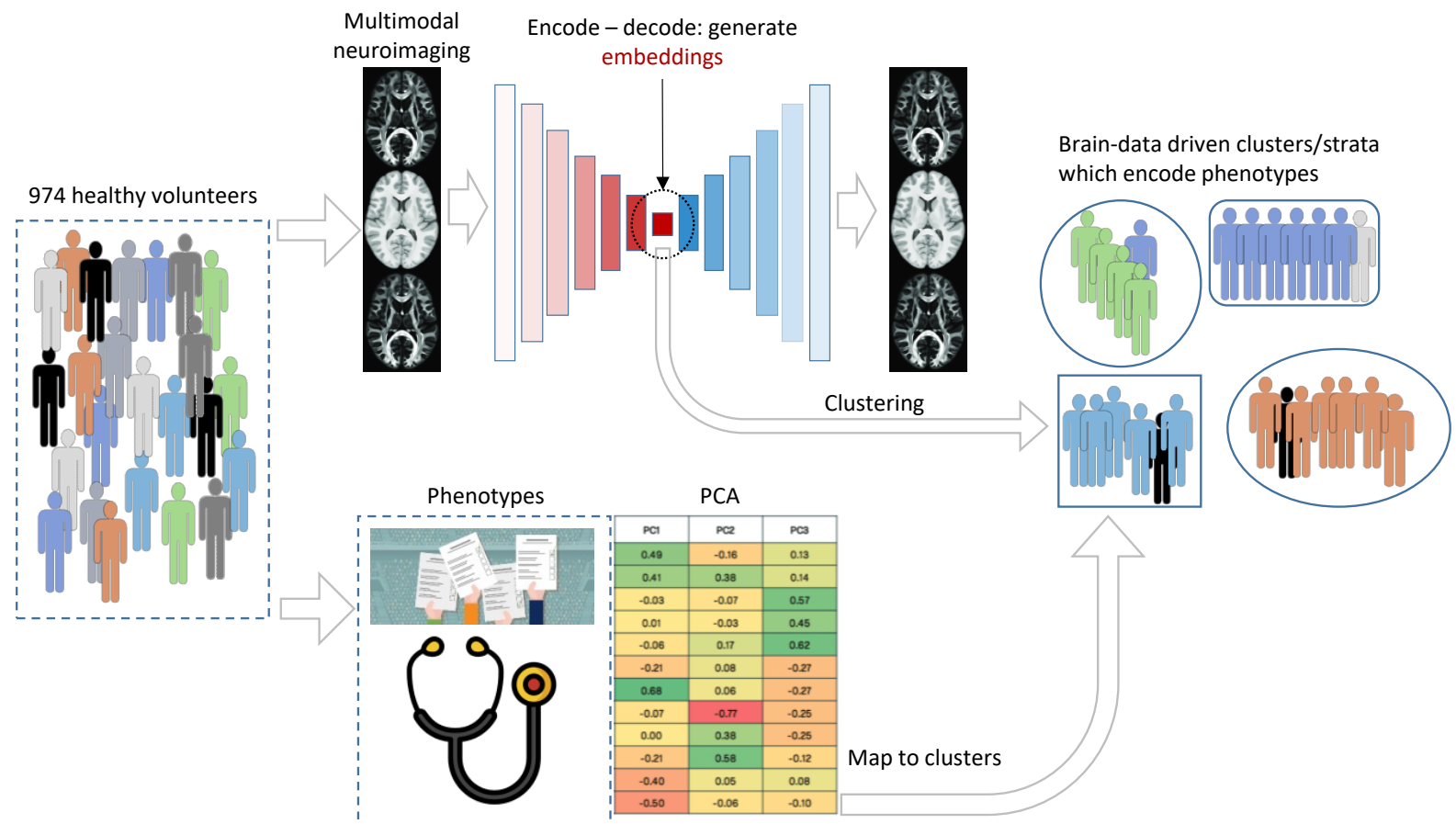

Figure 1: A summary of the workflow of our paper. First, we design an efficient multi-modal deep learning architecture to encode subjects in low dimensional embeddings. Our model is unsupervised, which means that it is trained to reconstruct the original inputs. The learnt embeddings summarize information from multiple modalities. Then, the model upsamples the embeddings to generate a separate reconstruction for each input modality, using information from both modalities. Second, we cluster the subject embeddings using affinity propagation to stratify the subject population by identifying separate subtypes/subgroups. Third, we aim to demonstrate the external validity to our framework by mapping each of the identified clusters with external phenotypic traits/factors (which have been generated from a number of raw phenotypic scores and interpreted by an expert neuroscientist, LP) which did not concur to forming the embeddings. This is done by simply averaging the factor loadings within each cluster for each factor. This results in clustering the population using only brain imaging data. Clusters are then mapped to each of the factors.

\subsection{Multi-Modal Deep Learning architecture}

\subsubsection{Overview}

The architecture designed for this study can be viewed as a deep learning, unsupervised autoencoder, composed of a downsampling stage (encoder) and an upsampling stage (decoder) (See Fig 2). Several details are inspired from our recent supervised dual learning framework designed to process 3D medical images (Spasov et al., 2019). Here, on top of adopting an encode-decode design, in order to improve granularity, we incorporate skip connections between operational blocks at both stages (Ronneberger, et al 2015). The key aspects of our design choices can be summarized as: A) Parameter efficiency: in addition to conventional convolutional layers, we employed alternating sequences of depth-wise and pointwise convolutions, also known as depth-wise separable convolutions (F. Chollet 2017, A.G. Howard et al. 2017). This results in a lightweight neural network architecture with high parameter efficiency. This strategy has achieved superior performance in classification as well as image segmentation tasks (F. Chollet 2017, A.G. Howard et al. 2017, Zoph et al. 2018) and has been extended to medical image classification (Spasov et al. 2019), while limiting overfitting. B) Multi-modality: it is well established that combining various medical imaging modalities can greatly enhance diagnostic 
and prognostic performance. We incorporate this data fusion aspect by employing a separate encoder and decoder for each of the modalities, which then all concur to generate a common embedding. The embedded representation is therefore learned (and contains information) from all modalities and can also be used for downstream learning tasks (see Section 3.2. and thereafter for a proof-of-concept experiment on multimodal neuroimaging data). Crucially, the decode step is based on this multimodal embedding only, demonstrating that modality-specific information can be re-distilled from the fused,

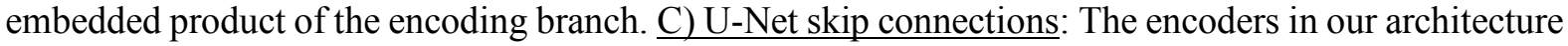
reduce image dimensionality by consecutively pooling the output features of previous layers. This loss of resolution poses a difficulty when learning to reconstruct high-fidelity images with good granularity. U-Net (Ronneberger, et al 2015) overcomes this challenge by supplementing the encoder-decoder architecture with contracting paths (or skip connections) between layers in the downsampling and layers in the upsampling stages. In this way we transfer localized high-resolution information directly to the reconstruction phase.

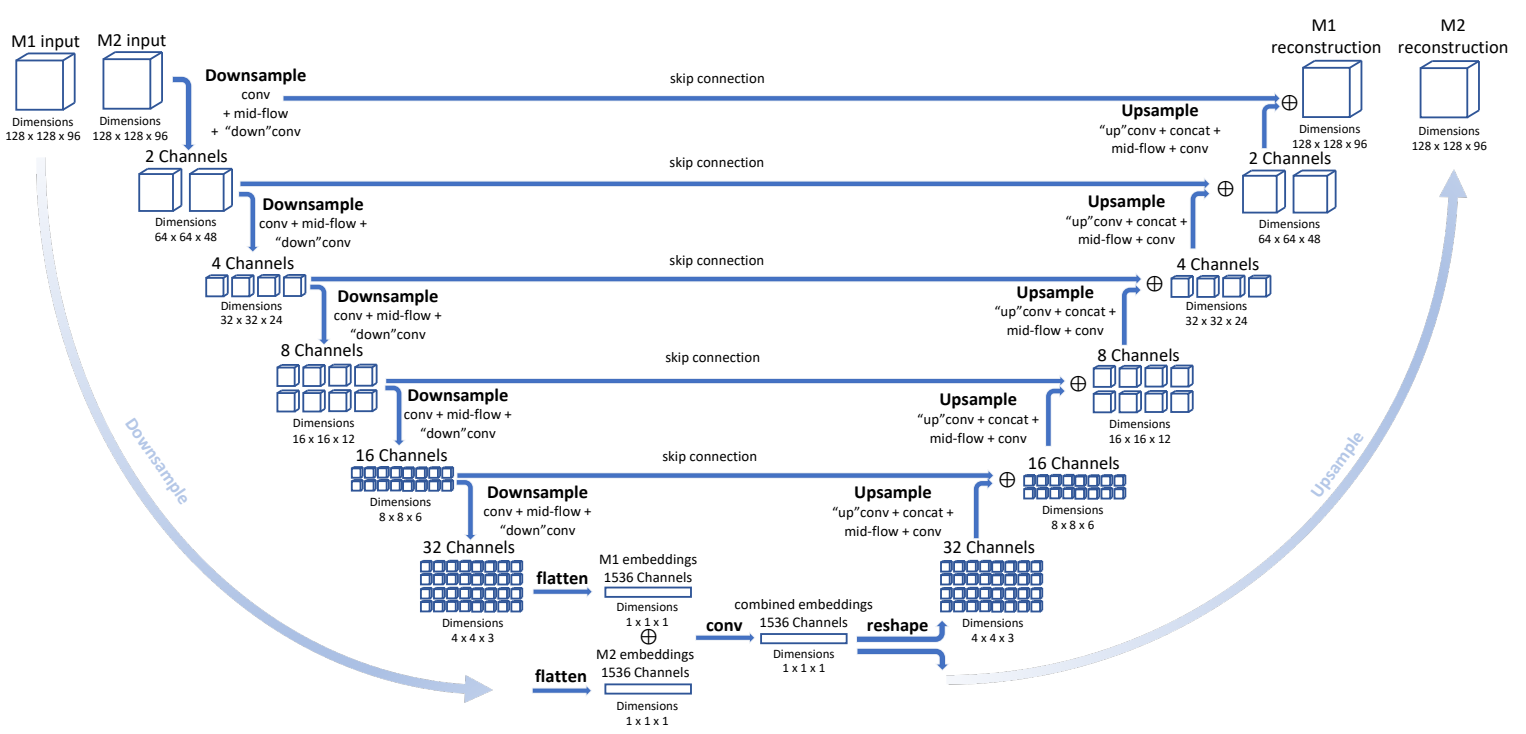

Figure 2: Our multi-modal architecture for unsupervised learning. The network simultaneously takes multiple modalities (two in this diagram, M1 and M2) and reconstructs both original inputs from a jointly learnt low dimensional embedding, denoted as "combined embeddings" in the figure. All modalities are processed in the downsampling and upsampling stages by successive applications of ad-hoc operational blocks termed conv, midflow, "down"conv and "up"conv. We reduce the dimensionality of the inputs via downsampling, and we merge their representations via concatenation $(\bigoplus$ symbol) and convolution. Then, each upsampling stage can be viewed as a mirror image of a downsampling stage at a certain depth. We concatenate corresponding feature maps between the upsampling and downsampling stages, and apply a series of "up'conv, mid-flow and conv block operations to recover the original dimensionality of the inputs. Network parameters are learnt by minimizing the binary cross-entropy between the reconstructions and input images.

\subsubsection{Architecture details}

Figure 2 depicts the network architecture of our deep learning autoencoder for medical image embedding and reconstruction (two modalities in the diagram). All modalities have a single channel and voxel values are scaled in the $[0,1]$ range prior to processing. In this paper, all training was performed with two modalities (M1 and M2, see Section 2.3 for data details, possible combinations and Section 3. for realistic examples), where each input was a 3D image of size $128 \times 128 \times 96$, resulting in approximately $12.2 \mathrm{M}$ data points per modality. The joint multimodal embedding vector is composed of 1536 elements (hence representing an approximately 16000-fold data reduction in the encode step) end employed to recover the initial dimensionality, contrast and information of the inputs. Both the encode and decode stages are implemented as a sequence of operational blocks termed conv, mid-flow, "down"conv and "up"conv (see below). The parameter-efficient separable convolutions (Spasov et al 2019) are integrated within the mid-flow block and comprise the majority of convolutional procedures 
in the network. At each downsampling step we apply a sequence of operations comprising "conv", midflow and "down"conv, and during upsampling - "up"conv, concat (concatenation needed for skip connections), mid-flow and conv. The inner working of these operational blocks is depicted in Fig. 3. In the downsampling stage we consecutively reduce the dimensionality of the inputs in 5 steps. At each step each dimension of the 3D images is halved, and the number of convolutional filters is doubled. We undo this process during upsampling by upscaling the embeddings by the same factor of 2 at each step. The downsampling and upsampling streams process all modalities in parallel. We only combine the low-dimensional representations of each input by first flattening them separately, then concatenating (denoted by $\oplus$ symbol in Fig. 2) and convolving them. To illustrate how high granularity is achieved through skip connections, consider the output feature maps with dimensions $32 \times 32 \times 24$ and 4 channels in the upsampling stage. First, we "up"conv these activations and scale each dimension twice to $64 \times 64 \times 48$. Then, we concatenate these intermediate representations with feature maps produced after a single step of downsampling, which have the same dimensionality, as denoted by the directed arrow labelled "skip connection" in Fig. 2. We then mix the combined representations with a mid-flow and a conv block to produce the upsampled feature maps one level higher in the Upsample stream (dimensions $64 \times 64 \times 48$ with 2 channels).

\subsubsection{Operational blocks}

In both the encoding and decoding stages, we employ a series of operational blocks, similar to the ones proposed in Spasov et al., 2019, comprising conv, "up"conv, "down"conv, mid-flow (see Fig. 2 for a schematic scheme of the whole DL network). Fig. 3 depicts the most foundational block in our neural network model - the (sep)conv operational block. It has two variants - one working with a standard 3D convolutional operator (conv) and another utilizing 3D separable convolutions (sep conv). In both variants the inputs are first convolved, then batch normalization (Ioffe and Szegedy 2015) and an exponential linear unit activation are sequentially applied. We also allow for dropout (Srivastava, et al 2014) to be used as the last layer in the (sep)conv block. Both "down"conv and "up"conv are simple extensions of the conv block. For "down" conv we append 3D Max Pooling ( $($ ize $=3$, stride $=1$ ) to reduce the dimensionality of feature maps in the downsampling stream after conv block processing. On the other hand, in "up"conv, we prepend 3D upsampling (size doubles in each dimension) to the conv block. The majority of convolutional processing occurs in an operational block termed mid-flow (see Fig 2. b), which has two parallel lines of processing. On one hand, three consecutive sep convs are applied to the inputs. On the other, we introduce a skip connection which does not transform the inputs and allows them to propagate unchanged to the output, which has been proposed to facilitate training of deeper neural networks (He et al., 2016). Finally, we combine the parallel lines of processing by adding them and returning a single output feature map. Substituting standard convolutions with separable ones results in a 20 -fold decrease in parameter utilization in a single mid-flow block. A thorough presentation of separable convolutions and their functional difference from standard convolutions is given in $\mathrm{F}$. Chollet, 2017 and their implication to parameter efficiency in medical imaging application is discussed in Spasov et al., 2019.

The model was implemented in Keras, with a TensorFlow backend and trained on a Nvidia TITAN V GPU. The training was performed over 200 epochs, batch size 1 . We set the dropout rate at 0.1 for all conv and sep conv operational blocks and apply an L2 regularization coefficient of $5 \times 10^{-5}$ for all network parameters. We use the Adam optimizer (Kingma et al., 2014) at its default settings to train the neural network. 
(sep)conv block inputs

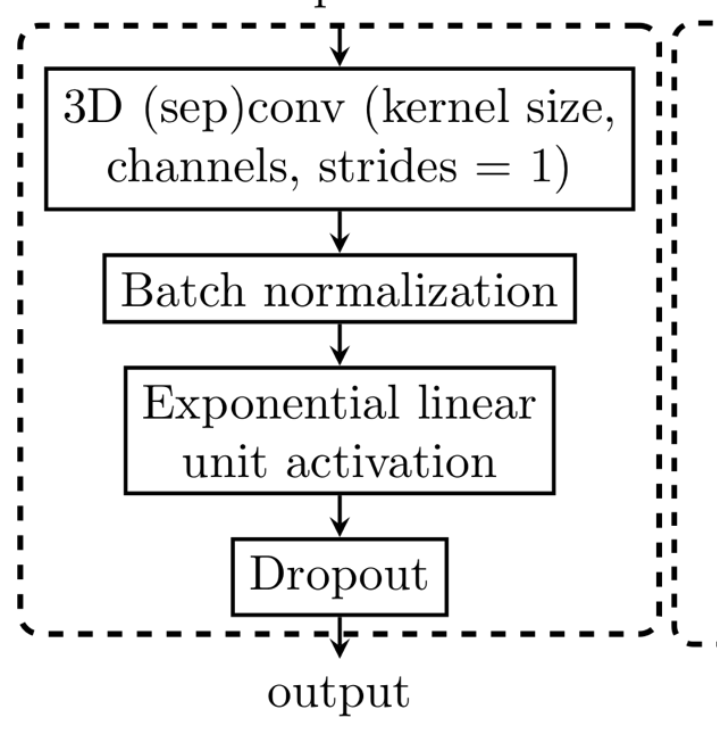

a) mid-flow block

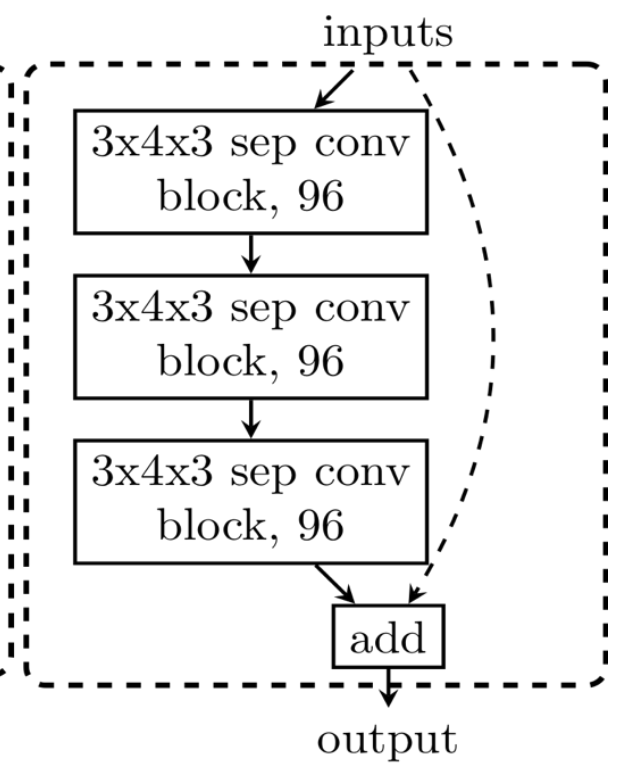

b)

Figure 3: Inner workings of the fundamental blocks used in the neural network diagram from Fig. 2. Since the conv, sep conv, "down" conv and "up" conv blocks are very similar in structure, we present a single diagram in fig 2. a) and use it to describe the other derived operational blocks. For example, in "down" conv we apply $3 D$ Max Pooling (size=3, stride=1) at the end of processing, whereas in "up" conv, we prepend 3D Upsampling (size doubles in each dimension); sep conv denotes using separable instead of conventional convolutions. Fig 1. b) presents the mid-flow block based on a series of three sep conv blocks and a skip connection adding the original input and the output of the final separable convolution. All convolutional kernel sizes in our work are of size $5 \times 6 \times 5$ with stride $=1$. The number of channels can be inferred from Fig. 2.

\subsection{Cross-Validation Procedure and Assessment of the reconstruction quality}

In order to evaluate the performance of our encoder-decoder architecture, and verify our model did not suffer from overfitting, we implemented a 10 -fold cross validation procedure using two modalities from our data set of approximately 1000 subjects. The following procedures / metrics were employed for each fold to assess the "difference" between the original and reconstructed images, and hence the overall reconstruction performance.

1) Mean Squared Error (MSE) computation. The MSE between each reconstructed and original pair was computed across whole voxelwise images.

2) Definition of a measure called henceforth "normalized difference" (NormDiff), defined as NormDiff $=\frac{\text { reconstructed-real }}{\text { reconstructed }+ \text { real }}$. This metric is bounded between -1 and 1 , and is intended to quantify asymmetry between a real and a reconstructed value. The advantage of this is to eliminate dependency on the underlying image intensity and to focus on relative errors. By calculating this metric voxel-wise, we obtained normalized difference images which are then collapsed through the median operator to obtain one value for each real-reconstructed pair.

3) Estimation of Contrast to noise ratio (CNR), an important metric in diagnostic imaging. We adopted a simple definition of CNR based on two randomly selected regions of interest (ROI), namely $C N R_{1,2}=$ $\frac{m e a n(R O I 1)-m e a n(R O I 2)}{s t d(R O I 1+R O I 2)}$. We then divided each image in regular regions of interest (ROIs) of dimension $4 \times 4 \times 3$, and successively randomly sampled 1000 ROI pairs while avoiding sampling from the background. This yielded a 1000-sample estimate of the CNR distribution of each image, which was collapsed through the median operator to obtain one value for each real image and one value for each corresponding reconstructed image. Finally, the reconstructed-real difference in estimated median CNR was evaluated using the normalized difference as defined above. 


\subsection{Data and pre-processing}

\subsubsection{Population}

The population used for the present study consists in 974 subjects drawn from the publicly available Human Connectome Project (HCP) public data repository located at https://humanconnectome.org/ (see Table 1 for demographics). The cohort resulted from down selecting the total 1200 subject to those who had valid and downloadable diffusion MRI data (see Table 1) at the time of processing. Additional phenotypic variables are summarized in the supplementary materials (Table S1).

\begin{tabular}{l|lll}
\multicolumn{2}{c}{ Age } & Handedness & BMI \\
\hline $\begin{array}{l}\text { Males } \\
(n=444)\end{array}$ & $27.94 \pm 3.65$ & $60.54 \pm 43.63$ & $26.78 \pm 4.36$ \\
$\begin{array}{c}\text { Females } \\
(n=530)\end{array}$ & $29.42 \pm 3.52$ & $69.88 \pm 43.93$ & $26.10 \pm 5.85$
\end{tabular}

Table 1: The table shows median and standard deviation of three demographics information on the HCP cohort studied: age, handedness and BMI $(n=974)$

\subsubsection{Phenotypic Variables and factor analysis}

In order to better characterize our study population, and also in order to test the ability of the information encoded in our multimodal embeddings to map to distinct phenotypic groups, we clustered the population in the latent embeddings space. For this purpose, 51 questionnaire measures, demographic variables, or neuropsychological tests assessing for lifestyle, psychological and physical well-being, personality, cognitive functioning, and emotional behavior were included in this study (see Supplementary material). A principal component analysis (PCA) with Varimax rotation was used to reduce data dimensionality and identify the main factors for the psychological, cognitive, and physical and mental health latent dimensions. Varimax rotation rotates the orthogonal basis to dispersion of the loading scores across components, hence simplifying the expression of the subspace extracted. The rotated component matrix was employed for extraction of individual factor (retaining only factors which corresponded to eigenvalues $>1$ ) loading scores using the regression methods. Those scores were then employed for characterizing the strata generated by unsupervised clustering of our multimodal embeddings (see Section 2.4). In order to provide an operational neurobiological interpretation for each factor, ladings were thresholded at an absolute value of 0.3 , which allowed an expert neuroscientist (LP) to identify the underlying factors and interpret them according to common associations between phenotypic variables.

\subsubsection{MR data acquisition}

All imaging data employed in this study were acquired by the HCP consortium on a Siemens Skyra 3T scanner with a customized SC72 gradient insert. T1w 3D MPRAGE images were acquired with $\mathrm{TR}=2400 \mathrm{~ms}, \mathrm{TE}=2.14 \mathrm{~ms}, \mathrm{TI}=1000 \mathrm{~ms}$, flip angle $=8 \mathrm{deg}, \mathrm{FOV}=224 \times 224,0.7 \mathrm{~mm}$ isotropic voxel, bandwidth $=210 \mathrm{~Hz} / \mathrm{px}$, iPAT $=2$, Acquisition time $=7: 40$ (min:sec). Diffusion weighted images were acquired with Spin-echo EPI sequences (b-values = 0, 1000, 2000, $3000 \mathrm{~s} / \mathrm{mm} 2$ in 90 gradient directions (interspersed with an approximately equal number of acquisitions on each shell). Diffusion weighting consisted of 3 shells of $b=1000,2000$, and $3000 \mathrm{~s} / \mathrm{mm} 2$. The diffusion directions were uniformly distributed in multiple q-space shells and optimized so that every subset of the first $\mathrm{M}$ directions is also isotropic (Caruver et al., 2013), $\mathrm{TR}=5520 \mathrm{~ms}, \mathrm{TE}=89.5 \mathrm{~ms}$, flip angle $=78 \mathrm{deg}$, refocusing flip angle $=160 \mathrm{deg}, \mathrm{FOV}=210 \times 180(\mathrm{RO} \times \mathrm{PE})$ matrix $=168 \times 144$ (RO $\times$ PE), slice thickness $=1.25 \mathrm{~mm}, 111$ slices, $1.25 \mathrm{~mm}$ isotropic voxels, Multiband factor $=3$, Echo spacing $=0.78 \mathrm{~ms}, \mathrm{BW}=1488 \mathrm{~Hz} / \mathrm{Px}$, Phase partial Fourier 6/8). A full diffusion MRI session included 6 runs (approximately 9 minutes and 50 
seconds each). Diffusion gradients were monopolar. Image reconstruction uses SENSE multi-channel (Sotiropoulos et al., 2013).

See

https://humanconnectome.org/storage/app/media/documentation/s1200/HCP S1200_Release Referen ce Manual.pdf. for additional details. All brain images were reviewed for incidental brain abnormalities by a neuro-radiologist, and pre-processed following the minimal HCP pipeline by the HCP consortium (Glasser et al., 2013).

\subsubsection{Diffusion data pre-processing and model fitting}

Diffusion image preprocessing, performed by the HCP consortium, included state-of the art procedures: intensity normalization across runs, distortion correction through the 'TOPUP' tool (part of FSL, (Jenkinson et al., 2012), eddy current and motion correction through the 'EDDY' tool (also part of FSL), gradient nonlinearity correction, calculation of resulting gradient bvalue/bvector deviation, and Registration of mean $\mathrm{b} 0$ to the corresponding T1w volume with FLIRT BBR+bbregister (also part of FSL). This is followed by transformation of diffusion data, gradient deviation, and gradient directions to $1.25 \mathrm{~mm}$ structural $(\mathrm{T} 1 \mathrm{w})$ space. Starting from these preprocessed data both the tensor model (Diffusion Tensor Imaging - DTI, only bvalues $<=1000$ ) and the NODDI model (all data) (Zhang et al., 2012) were fitted to each individual dataset using the microstructure diffusion toolbox (MDT) (https://github.com/robbert-harms/MDT). From the DTI model, we extracted fractional anisotropy maps (FA), which are known to be sensitive to microstructural alterations. From the NODDI model we extracted neurite dispersion indices maps (NDI), which are known to be even more specific to the same type of alterations.

\subsubsection{Study specific template creation and image registration}

To improve registration accuracy, we built a study-specific T1 template using the T1-weighted images After bias filed correction in FSL, all T1-weighted images were non-linearly co-registered to each other (symmetrical diffeomorphic mapping), averaged iteratively (5 iterations), and co-registered to the final template. A binary brain mask was generated from the T1 template using BET2, also part of FSL.

The template creation and registration procedures were performed using the Advanced Normalization Tools (ANTs) software (Avants et al., 2011). Non-linear transformations were initialized through a chain of center of mass alignment, rigid, similarity, and fully affine transformations followed by local nonlinear warping (metric: neighborhood cross correlation, sampling: regular, gradient step size: 0.12 , four multi-resolution levels, smoothing sigmas: 3, 2, 1, 0 voxels in the reference image space, shrink factors: 6, 4, 2, 1 voxels, histogram matching, data winsorisation with quantiles: 0.001, 0.999, convergence when the slope of the normalized energy profile over the last 10 iterations $<10^{-8}$ ).

We then extracted the local Jacobian determinant (JAC) of the non-linear part of the last-stage deformation field which takes each T1-image into template space. The JD image quantifies the amount of local volume variation (contraction/expansion) computed when matching the single subject image to the template and is generated in the template space. Also, the same warp fields were applied to all diffusion derived maps (FA and NDI), resulting in all images (T1, FA, NDI, JAC) in the same $1.25 \mathrm{~mm}$ resolution space with high intra-modality and inter-subject anatomical correspondence and downsampled by a factor 2 to yield final volumes of size 128x128x96 (Figure 3). Finally, all images were robustly normalized into the $0-1$ range by using min-max normalization ( 0.1 and 99.9 percentile) across the whole population. Min-max normalization is often employed in deep learning to facilitate convergence of the learning algorithm. Image co-registration required $\sim 26,500$ hours of CPU time. Calculations were performed on a 600-node compute cluster with 8GB-RAM/node.

\subsection{Mapping multimodal embeddings to phenotypic variables}

\subsubsection{Clustering multimodal embeddings}


As proof of concept of the usefulness of our multimodal embedding in efficiently compressing information, we adopted an unsupervised clustering strategy in order to explore the existence of subgroups in the brain data (a near-impossible procedure when working directly with whole brain images) and mapped these subgroups onto the external phenotypic data which had not concurred to forming the embeddings. We employed affinity propagation (AP) clustering, which does not require an a priori number of clusters to be specified and performs well in the presence of noise (Vlasblom et al 2009). AP was used in conjunction with Euclidean Distances and relies on two main parameters: preference (which determines the likelihood of a point to be chosen as exemplar) and damping (related to the speed and accuracy of numerical stabilization). These were optimized through a grid search procedure across 10 damping (0.5-1, equal intervals) and 1000 preference values (sampled with equal spacing between bottom and top percentile of the negative squared Euclidean Distances of the data matrix at hand) using the Silhouette index score as a performance metric (Rousseeuw et al 1987).

The between-cluster differences in factors extracted from the phenotypic variables were assessed through the non-parametric Kruskall-Wallis tests (KW) (Wayne, 1990) and corrected for multiple comparisons across factors through the FDR Benjamini and Hockberg correction procedure (Benjamini $\&$ Hochberg, 1995). $\mathrm{P}<0.05$ (FDR corrected) was considered statistically significant.

\subsubsection{Bootstrap analysis between-cluster differences in phenotypical factors}

In order to test the hypothesis that the statistically significantly differences in phenotypic variables we found in the previous step may be a result of random effects, we proceeded as follows. Given $m$ subjects (i.e. embeddings) which have been partitioned into $n$ "true" clusters of sizes $s_{1} \ldots s_{n}$, as in 2.4.1, we randomly repartition the $m$ subjects in the clusters into same number of groups $(n)$ with the same sizes $\left(s_{1} \ldots s_{n}\right)$ as the "true" clusters and compute the corresponding KW statistics. This bootstrapping procedure is repeated $M$ times (here $M=10000$, sampling with replacement). We then evaluated the $p-$ value related to the hypothesis described above as the number of bootstraps whose KW statistics is higher than the "true" KW statistics, divided by M. This fraction of bootstraps serves as a surrogate pvalue corresponding to the above outlined hypothesis - i.e. if this pvalue is $<0.05$ (after FDR correction across all factors), the "true" statistical significance in clusterwise differences between factor values can be considered non-random.

\section{Results}

\subsection{Reconstruction results}

In order to evaluate the quality and generalization ability of our model, we performed experiments using three pairs of modalities i.e. JAC-FA, JAC-NDI, T1-NDI (Figure 4). These choices of pairs were made in order to a) be possibly sufficiently general to explore the ability of our architecture to combine different contrasts, and 2) generate pairs of modalities which, to some degree, complementarily included mainly white matter (WM) and grey matter (GM) contrast. The presence, in the full reconstructed images, of key anatomical detail was confirmed through sample whole-image inspection by an expert neurologist (LP). Visually, we obtained excellent reconstruction quality in FA, NDI and JAC images (Figure 4). Some loss of detail in the subcortical structures as imaged in the T1 contrast can be seen when T1 is paired with NDI. This is possibly due to overlapping anatomical redundancy with the NDI image. 

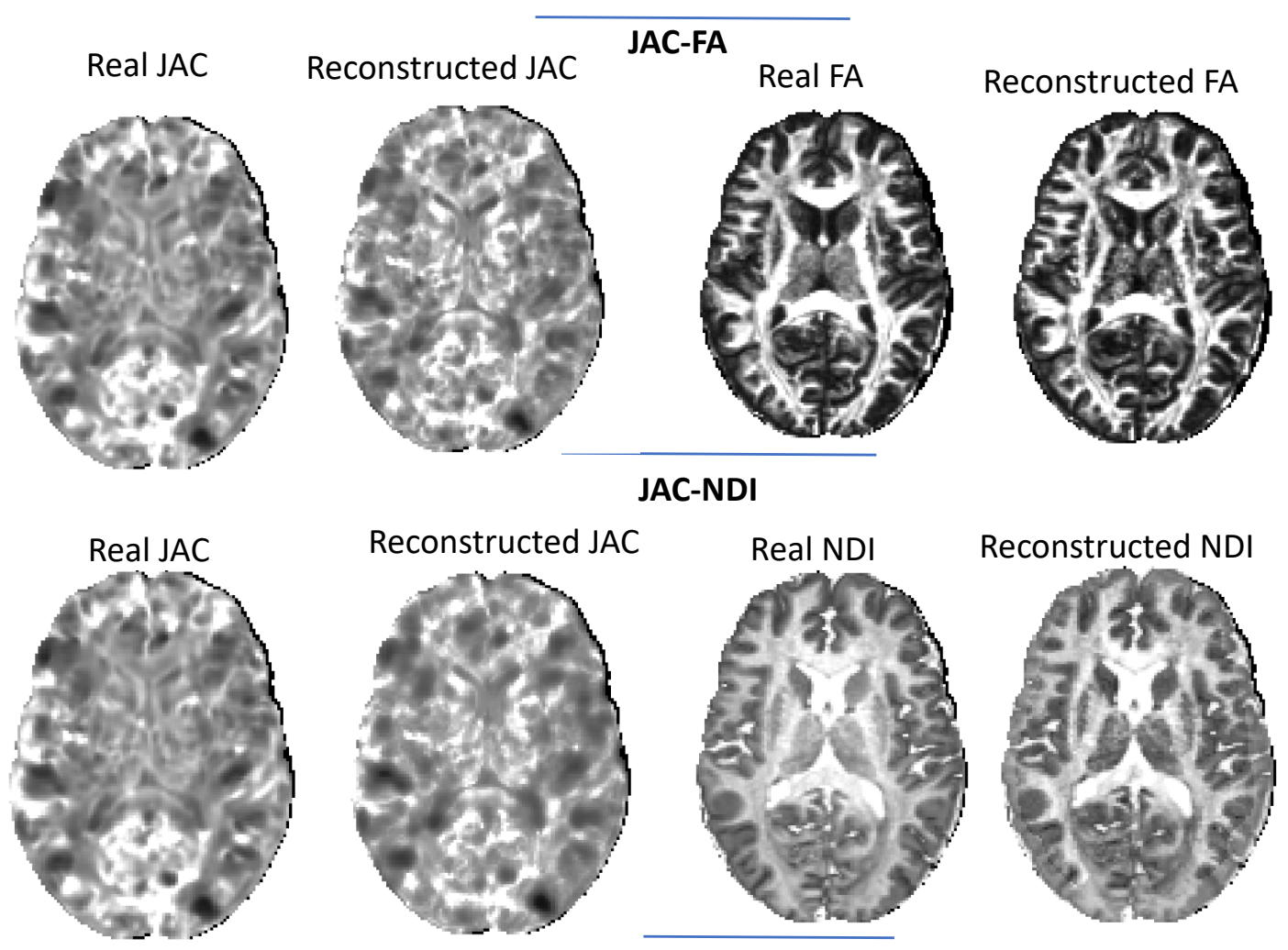

JAC-NDI
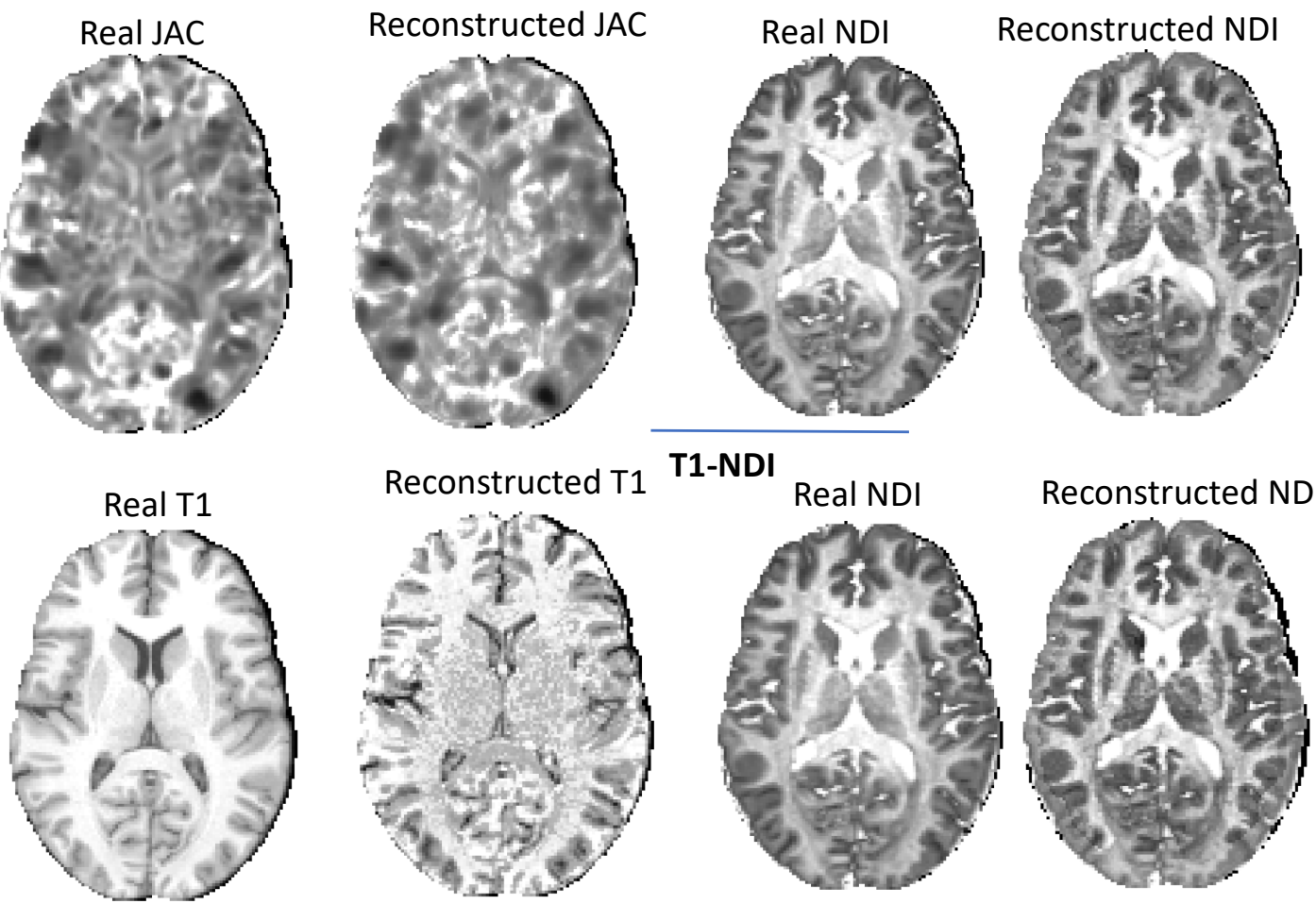

T1-NDI
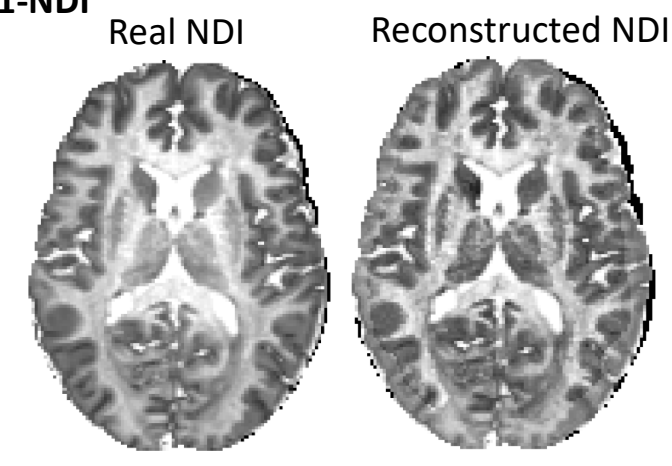

Figure 4: Example of pairs reconstructions for JAC-NDI, JAC-FA and T1-NDI modalities, using our multimodal architecture. Color-scales are arbitrary but equal for each real-reconstructed pair.

\subsection{Cross-validation results}

In this section, we present the cross-validation results (i.e. the generalization ability of our model) for one exemplary pair of modalities, namely JAC and NDI. This choice was made based on the facts that 1) the Jacobian determinant of a transformation which brings a T1 image into standard space has been seen to be a superior indicator of grey matter density changes as opposed to the T1 image intensities alone; 2) the NDI has shown promise and applicability in a vast number of neuroscience studies (along with a greater sensitivity and specificity in detecting microstructural alterations). Figure 5 depicts the 10 -fold cross-validation results as described in the Methods section. MSE was bounded between 0 and 0.02 in all folds, except for fold six where it reached a median value (across all subjects in the fold of approximately 0.038 ). Considering that both JD and NDI metrics are bounded by definition between 1,1 and 0,1 (respectively) and commonly assume absolute values up to $0.5-0.7$, these MSE values are around $1 / 200^{\text {th }}$ of the original intensities. 
CNR JAC

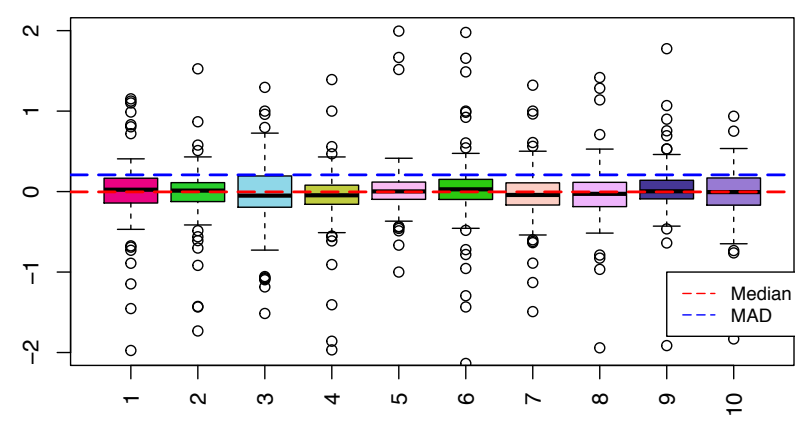

MSE (JAC)

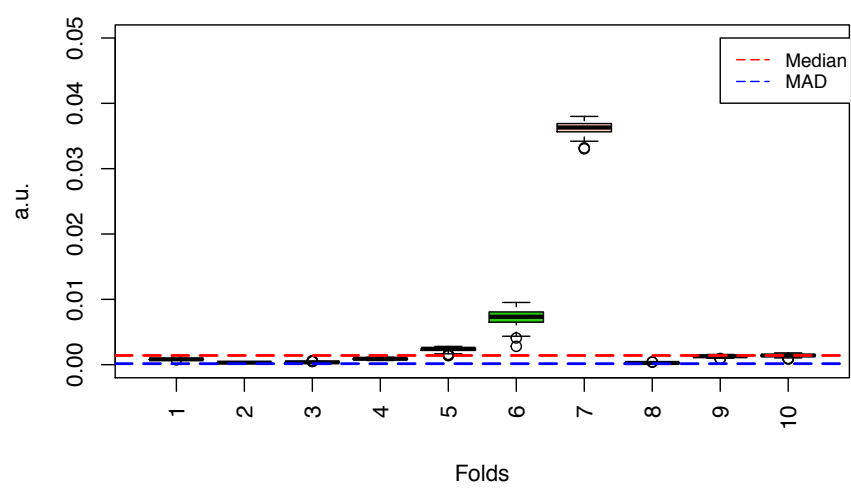

Normalized Differences (JAC)

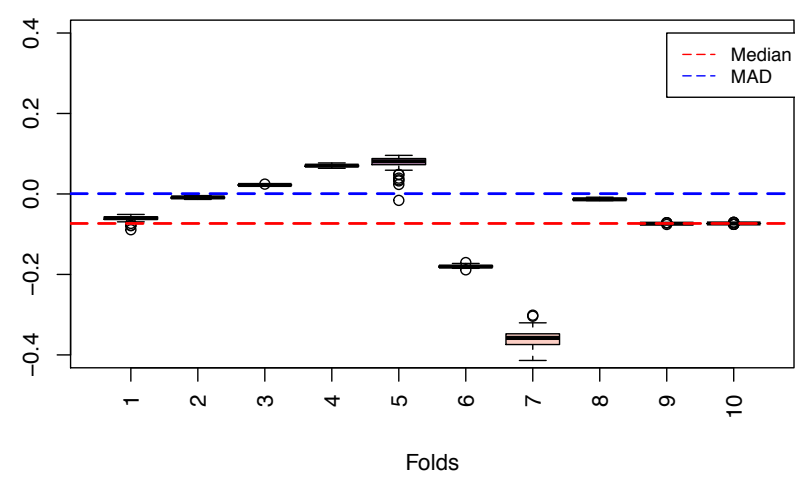

CNR NDI

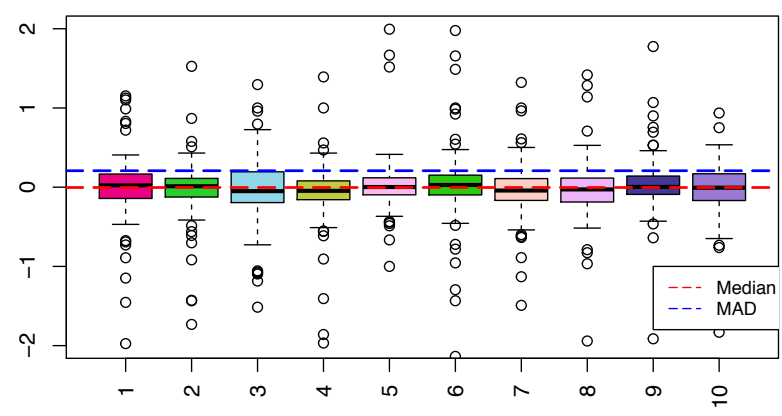

MSE (NDI)

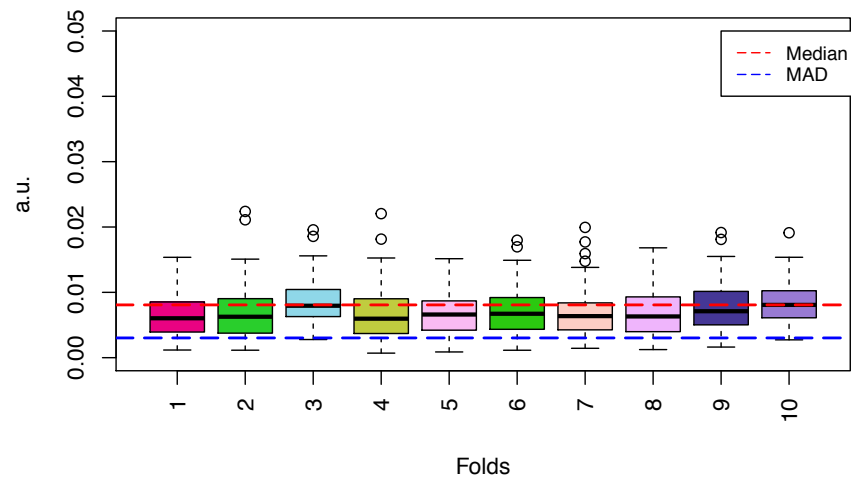

Normalized Differences (NDI)

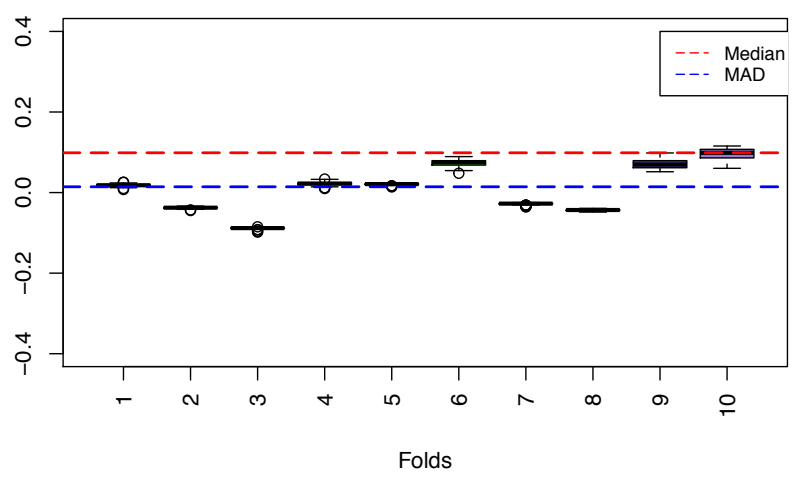

Figure 5: CNR, MSE and Normalized Differences for each of the 10 folds within the cross-validation procedure. The boxplots represent the distribution of the values, where the median and the interquantile ranges of the distribution are plotted. We also report the values of the Median (with the dotted red line) and of the Median Absolute Deviation (MAD, blue dotted line) across all folds. The $x$ axis represents the folds (from 1 to 10) and the $y$ axis the values of the respective plotted indicator.

\subsection{Phenotypic Variables and Factor Analysis}

The independent phenotypical dimensions derived from the PCA-based factor analysis (see Methods) resulted in retaining 13 factors which cumulatively explained $63 \%$ of the total variance (Figure 6). Upon inspection of the factor loadings by an expert neuroscientist (LP), we were able to assign proof-ofconcept, coherent interpretations to each factor based on the single phenotypic variables with highest loadings in each factor. In particular, in order of decreasing variance, these factors appeared to reflect negative affect; sociability; self-efficacy and wellbeing; crystallized intelligence; aggressive behavior; sustained attention and episodic memory; gender; fluid intelligence; high levels of blood pressure 
(hypertension); physical health/young age; positive personality traits (high agreeableness, high extraversion, and high mind openness); gait speed and physical endurance; right handedness.

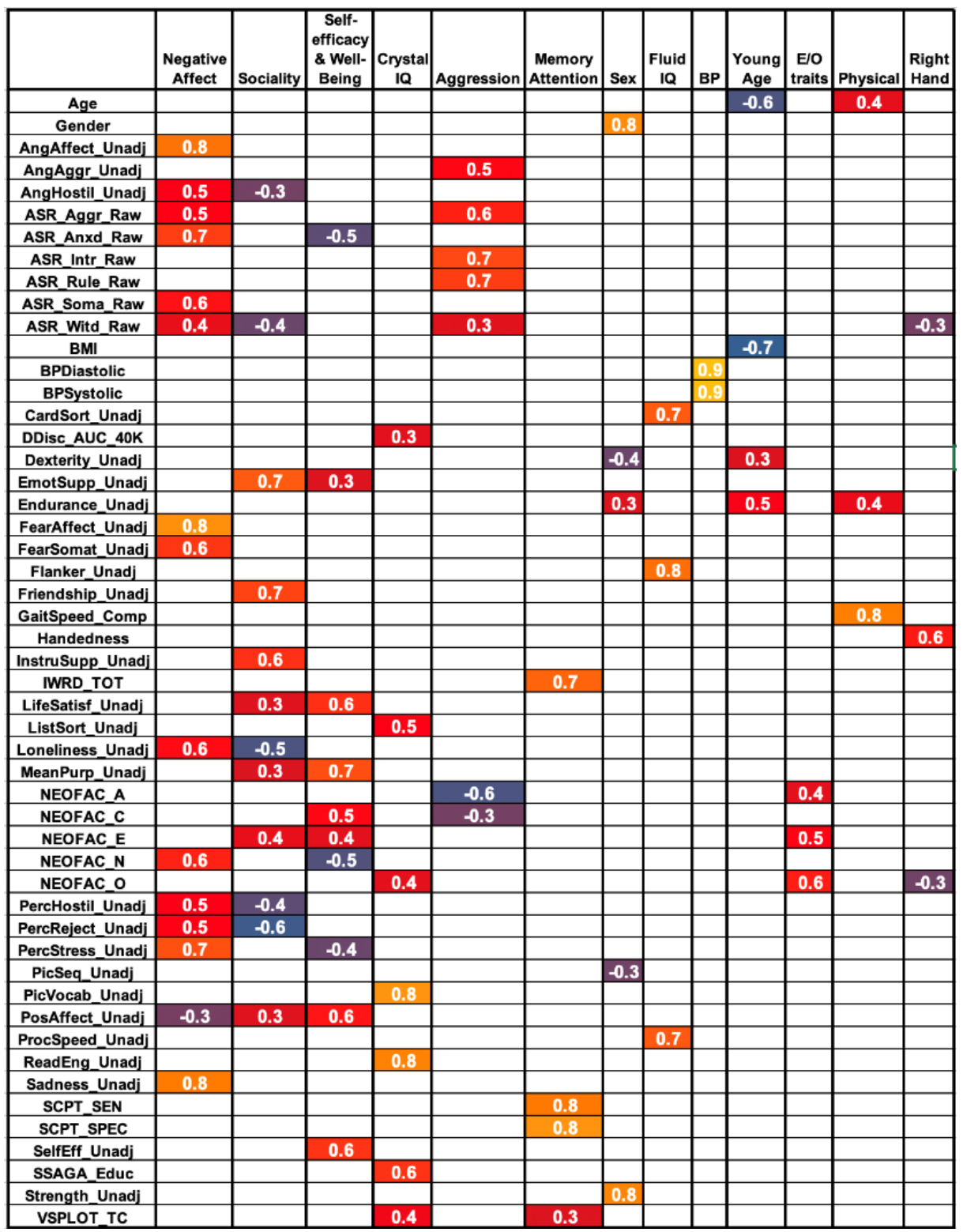

Figure 6. Loadings of each phenotypic variables (thresholded at an absolute value of 0.3 for visualization purposes) on each of the 13 factors which, cumulatively, explained $63 \%$ of the data variance. For a detailed explanation of the phenotypic variables and their origin, please see the HCP data dictionary at https://wiki.humanconnectome.org/display/PublicData/HCP+Data+Dictionary+Public-

+ Updated + for + the $+1200+$ Subject + Release. See supplementary materials for descriptive statistics of the original phenotypic variables. 


\subsection{Clustering and mapping multimodal embeddings to phenotypic variables}

When applying the clustering procedure to the multimodal embeddings computed (as described above) from the JAC and NDI images, we obtained nine distinct clusters (see Fig. 7 for sizes and for colour reference for subsequent results on phenotypic variables). Figure 7 shows the number of individuals belonging to each cluster for the JAC-NDI experiments performed.

\section{Cluster Sizes}

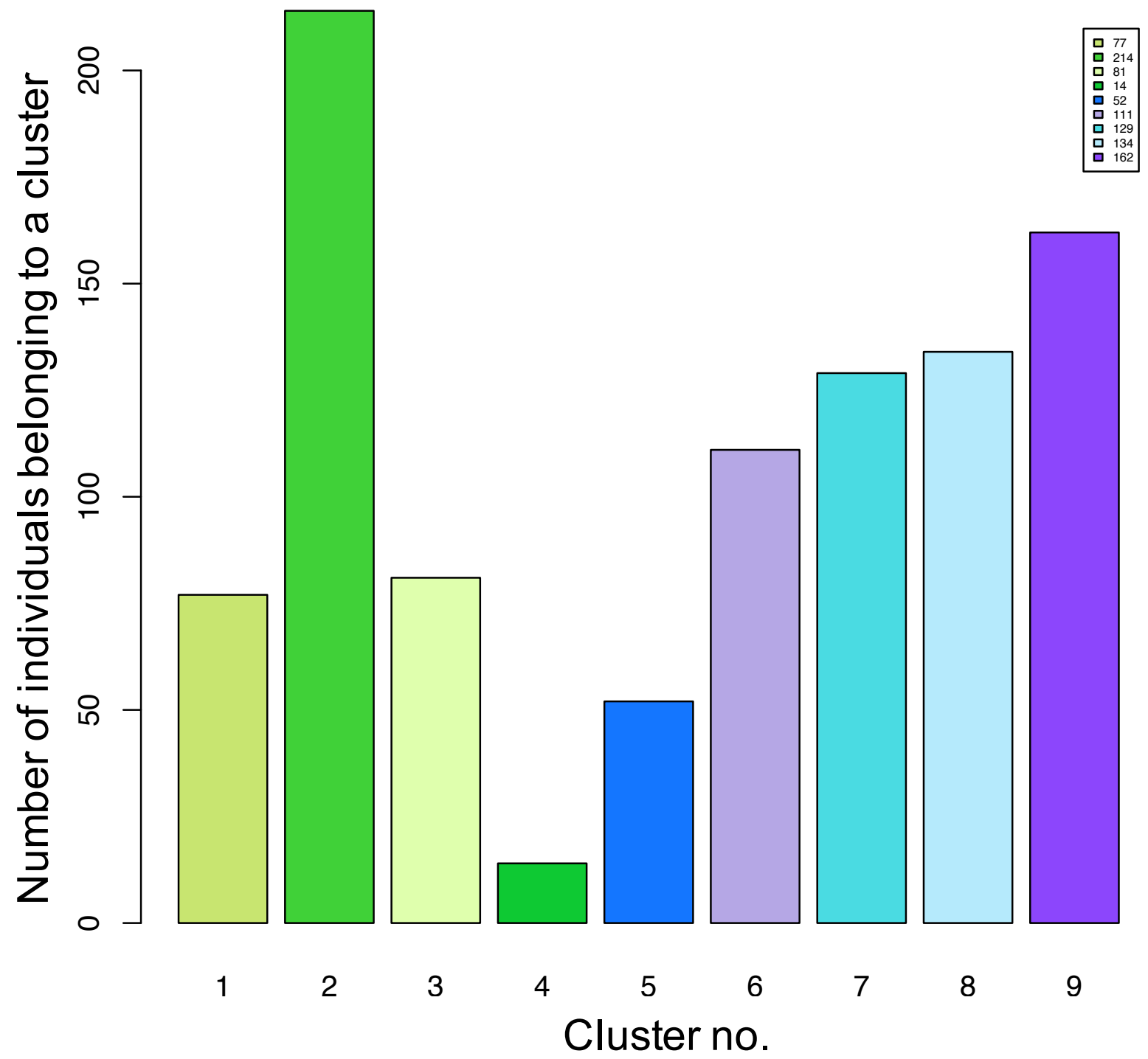

Figure 7: Clusters sizes obtained when running Affinity Propagation clustering with optimized parameters (see Methods) on the multimodal embeddings for the JAC-NDI embeddings

When using the KW test to explore between-cluster differences in factors extracted from the phenotypic variables, we obtained significant effects in six factors which had been identified as representing (in this sample) aggression, Blood Pressure, Crystallized Intelligence, Righthandedness, biological sex and Young age (FDR corrected across all factors), see Fig 8. In addition, for all these values, bootstrap analysis (see methods) showed that these effects (which were due to the assignment of subjects to each cluster of a certain size) were far not random (see Fig 9.). 

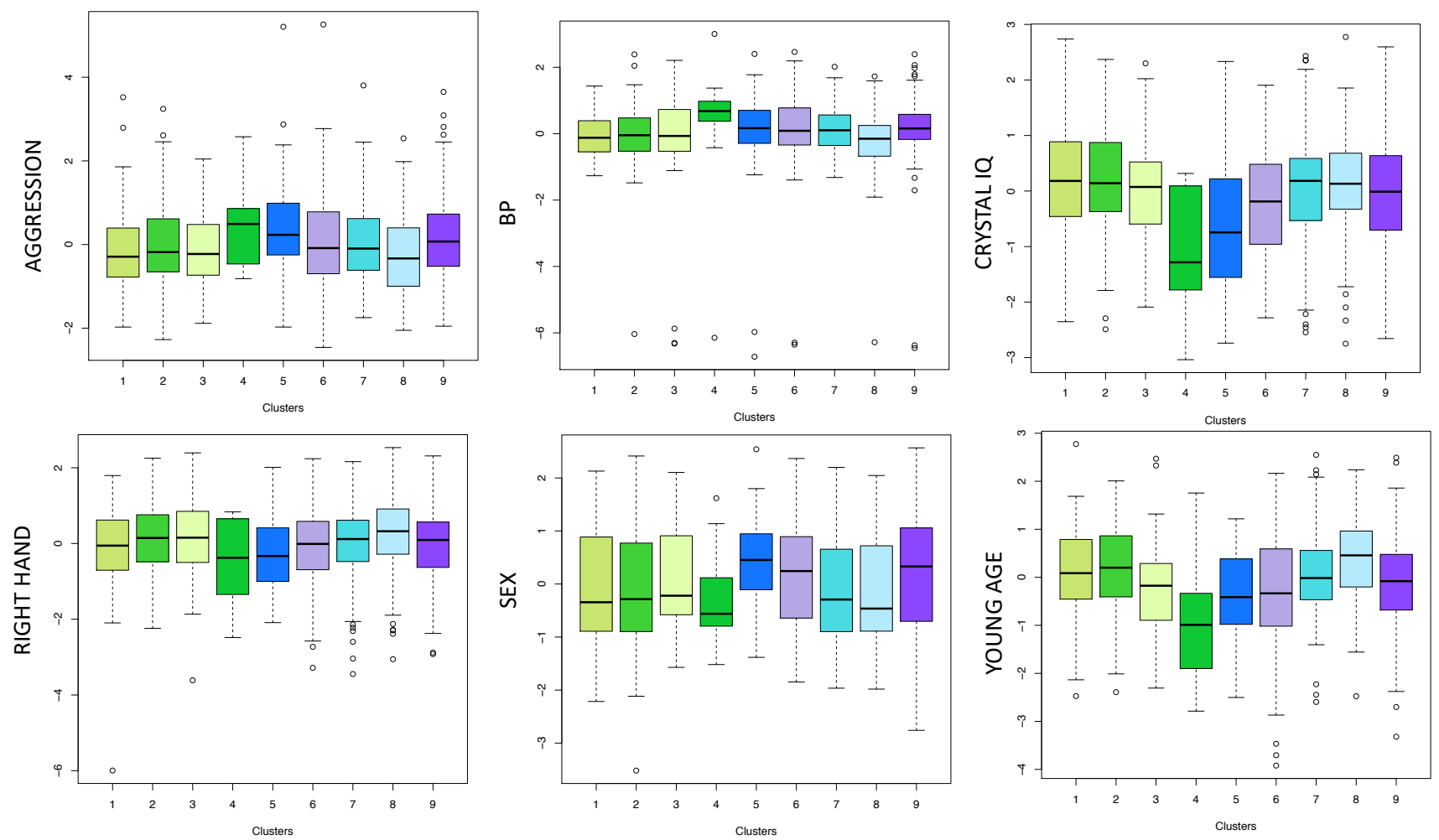

Figure 8: Phenotypic factor distribution across the nine identified clusters. Only phenotypic factors in which a significant difference (FDR corrected across clusters) across clusters are shown.

Aggression

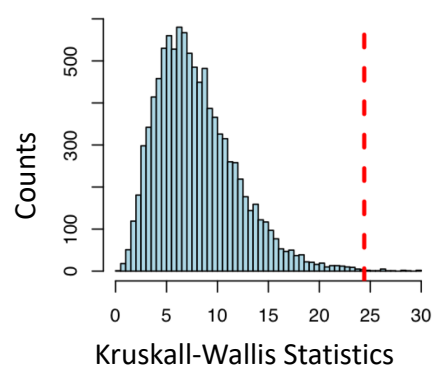

Crystal IQ

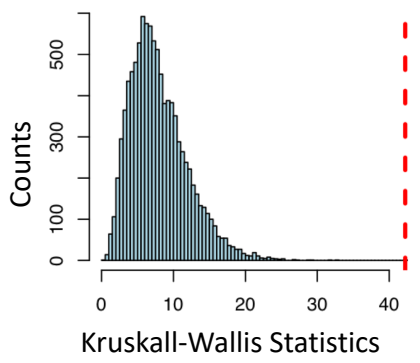

BP

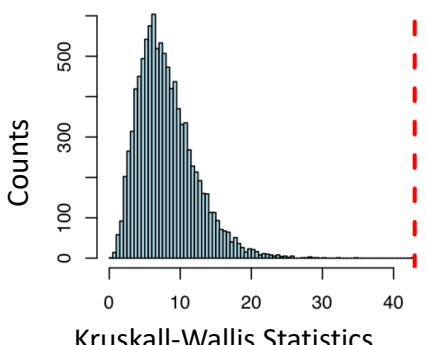

Kruskall-Wallis Statistics

Young Age

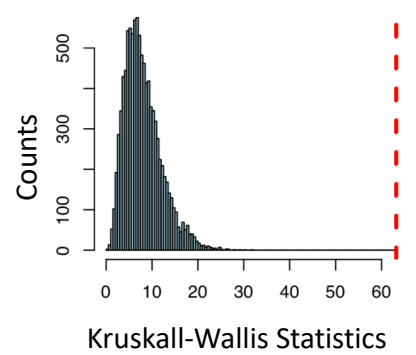

Right Hand

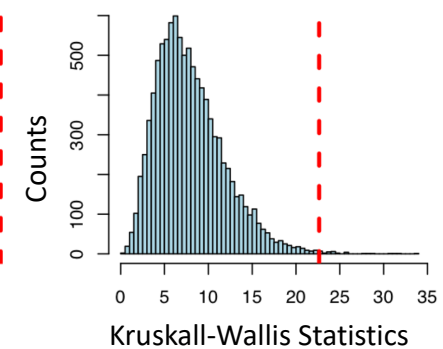

Sex

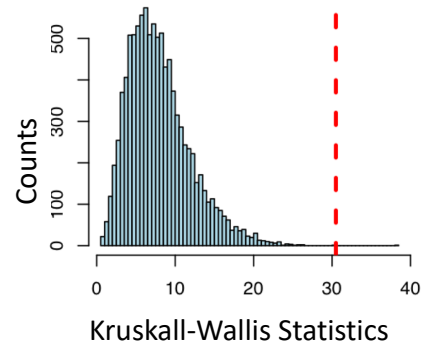

Figure 9: Bootstrap results comparing the "true" KW statistics (red, dotted line) resulting from our cluster assignments to the distribution of the KW statistics obtained when comparing Factor values between clusters after repeatedly (10000 samples) and randomly sampling individuals across cluster distributions with the same sizes. 
Finally, we asked the question whether the clusters we identified corresponded to recognizable/interpretable "profiles" (made of phenotypic factors) which could be further interpreted and/or heuristically explained. To this end, for each cluster and each factor, we computed the clusterwise median of each factor and evaluated its position (quantile) in the whole population. Figure 10 summarizes these results.

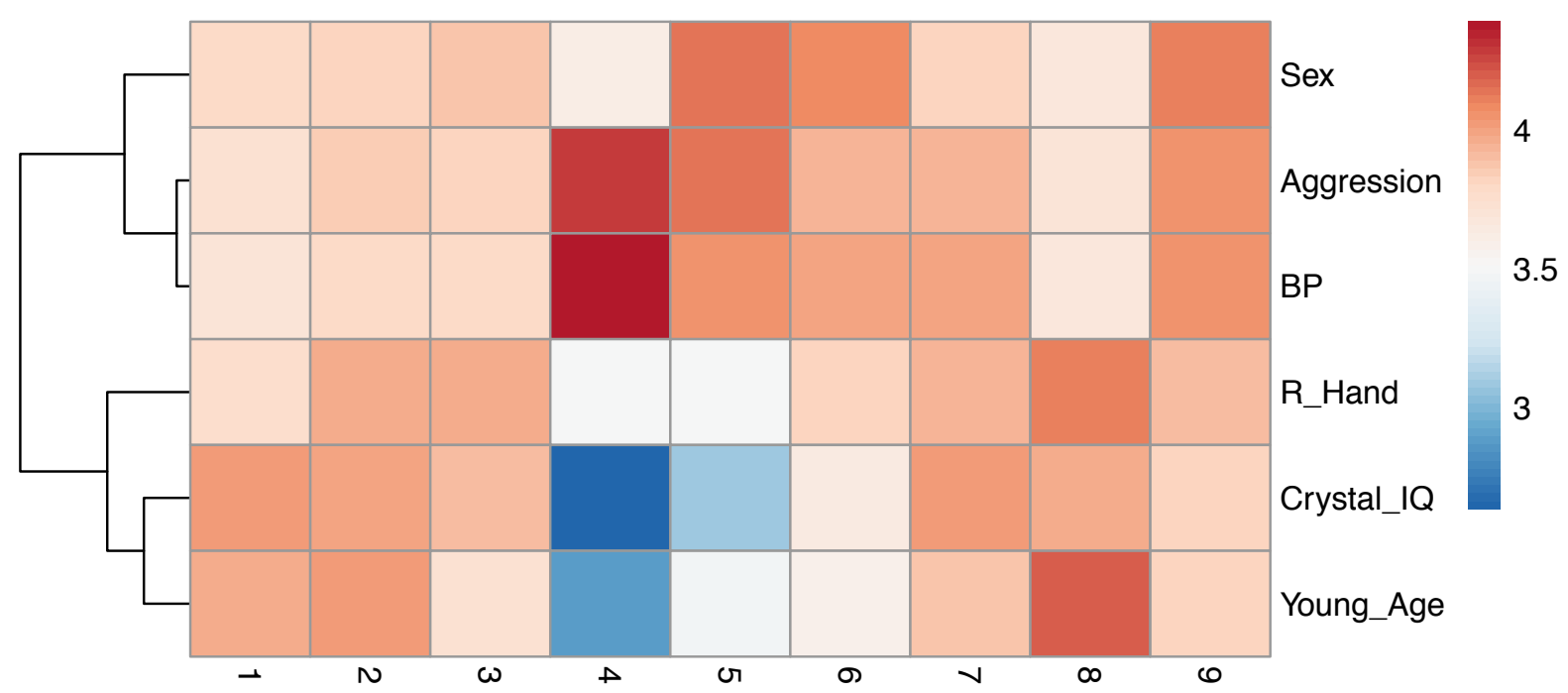

Figure 10: Cluster "profiles" Position (logarithm of the quantile of cluster-wise median of each factor with respect to the whole population distribution). Columns therefore represent cluster "profiles" as characterized by comparing their median value in each phenotypic factor to the distribution of the same factor across the population.

Interestingly, it is fairly evident how unsupervised clustering of our embeddings, generated from multimodal brain imaging only, not only yields separations which are non-random with respect to permuting individuals, but also that the clusters created have, to some extent, combinations of values of phenotypic traits which appear to delineate distinct, biologically consistent groups of individuals. An example would be cluster 4 composed by people with high blood pressure, high aggression, belowaverage crystal IQ and older age. While such an extreme cluster in hard to detect in a non-clinical sample of health people, it was picked up by our approach in spite of its small dimension (14 people). Cluster 5 appears to go in the same direction (albeit in a less extreme way), while being more polarized towards female sex.

\section{Discussion}

One of the main goals of this paper was to handle the extremely large amounts of information present in not only one, but multiple medical imaging modalities in a large number of subjects, maximizing information content and "compression" at the same time. Our framework is based on a modular approach and separable convolutional blocks, which can efficiently deal with highly dimension datasets. We aimed for the architecture to be generative in order to directly evaluate the decode part in terms of various reconstruction metrics. This ensures in turn that out low dimensional embeddings 1) succeed in encoding meaningful information, and 2) are able to regenerate the two modalities separately in spite of the complete information fusion implemented in the last encode step. Further, our deep learning architecture, which is heavily based on parameter-efficient separable convolutional blocks, is very lightweight, which reduces both training time and GPU memory utilization, therefore making our framework practical to handle multiple high-resolution 3D medical image modalities. In spite of the extremely large dimensionality reduction, we obtained excellent reconstruction quality in MSE, normalized differences and also CNR, an additional metric commonly used in evaluating the usefulness of medical images.

In this context, one notable prior study has presented an integrative analysis of features extracted from brain structural MRI, finding evidence for a subspace which is able to explain a large share of the 
inter-individual variation of brain structure in healthy people (Llera et al 2019). The authors also identified relationships between inter-individual brain structural variations and numerous demographic and behavioural variates, in particular demonstrating that a spectrum of behavioural and demographic data variability can be identified and explained using structural features only. However, the feature extraction method used in this paper requires a-priori information about which features to extract, hence strongly limiting generalizability. Other application of DL for dimensionality reduction in the field of medical imaging exist, see e.g. Benson et al., 2014 or Lundervold et al., 2019 for a review. However, most of these papers rely on a priori feature engineering like e.g. aggregating information by averaging within ROIs, hence heavily reducing the dimension of the problems fed to the actual DL architecture. Other methods rely on the extraction of patches (e.g. Saha et al., 2020, Kao et al., 2020) of a priori segmentation of white and grey matter volumes.

In our proof-of-concept application, we focused on an example pair of modalities, JAC and NDI. The Jacobian Determinant, or JD, is a voxel-wise measure of the volumetric changes that are computed from the deformation needed to precisely match each subject-specific structural MR image to a populationspecific MRI template. Relative to the more commonly used voxel based morphometry (VBM), tensorbased morphometry analyses derive JDs volumetric changes from non-linear warp fields, rather than from signal intensities alone (Avants et al., 2011; Chung et al., 2001; Villalon et al., 2011). In turn, the NDI has been associated with microscopic variability in the neurite architecture and geometry, highlighting the viability of the NDI as a proxy measure for biological changes in the neuropil (i.e. dendrites, axons, and glial filaments). The NDI is also considered a more sensitive diffusion-imaging metric compared to the canonical diffusion tensor imaging (DTI) imaging measures such as FA, MD, and RD. Notably, when clustering the embeddings in an unsupervised manner, we were able to demonstrate the creation of clusters based on brain data only, which map to differences in phenotypic dimensions. In addition to checking statistical significance of those differences, we also demonstrated through bootstrap analysis that those differences in phenotypic dimensions are not random with respect to the partitioning generated by the clustering algorithm. Also, when examining the phenotypic "profiles" of these clusters, in spite of the narrow age range (25-45) and, in general, great homogeneity of the HCP sample of healthy individuals (i.e. comparatively little variance to encode), we were able to detect both some expected and sensible associations, and some interesting, and novel characteristics of the cohort we stratified.

For example, cluster 4 grouped phenotypically 'extreme' male individuals with high levels of aggression, high blood pressure, relatively older age and lower crystallized IQ. Likewise, cluster 5 contains female participants displaying similar demographic, health, and behavioural features as those in cluster 4 (particularly the association between aggression and low IQ). Although the number of individuals grouped within each of these empirically derived categories is relatively low $(\mathrm{n}=14$ for cluster 4 and $n=52$ for cluster 5), the association between aggression and low IQ is interesting and expected on the basis of previous literature. More specifically, it highlights the proof-of-concept ability of our architecture to detect relevant phenotypic associations in non-clinical samples even when they exist only in a small subset of subjects. We feel that, in addition to heuristically validating the information content of our multimodal embeddings, these results demonstrate the possibility of a brainto-phenotype mapping which can easily be extended to the important application of identifying pathological substrates for phenotypic disturbances. Relatedly, in (Llera et al 2018) the authors present a comprehensive analysis of features extracted from brain MRI, features, linking the inter-individual brain structure variability to a large set of behavioural and demographics scores. Again however, in this paper the features are extracted a-priori from the input dataset and are not learnt during training, rendering this method application-specific. Numerous examples in the literature demonstrate the usefulness of unsupervised approaches to stratifying patients such as cancer, or neurodegenerative pathologies as for example Alzheimer (Lopez et al 2018, Lasko et al 2013, Wang et al 2013, Wu et al 2017, Alashwal et al 2019, Young et al 2017). Similar studies allowed to predict patient evolution based on clusters generated from both cerebrospinal fluid MRI data (Nettiksimmons et al., 2010). Also, previous work clustered $\mathrm{AD}$ patients using, e.g., the CLustering In QUEst (CLIQUE) strategy (Gamberger et al., 2016a, 2016b), hierarchical clustering or k-means clustering for profiling AD patients, older adults in general (Escudero et al., 2012; Zemedikun et al., 2018), or patients with other 
disorders, including aphasia (Hoffman et al., 2017). Again, all these studies rely on some sort of prior engineering which pre-emptively eliminated the need for learning data reduction during training. Also, initial efforts have been made towards a biomarker-based characterization of substrata of neurological disease. For example, the so-called A/T/N categorization is a simple conceptual clinical and biological construct which may, to some extent, stratify the pathophysiological continuum of AD and other neurodegenerative disorders according to the presence of established and reproducible AD-related pathophysiological markers (Bennett et al., 2016). Still, from a machine learning perspective, in this type of procedure the feature engineering and learning steps are disjoint (hence sacrificing representational ability) and the generalizability of such categories as well as the optimality of the cutoffs employed in generating them has not been investigated. Unbiased stratification of the AD continuum was also explored using CSF markers alone (Toschi et al., 2019). Similar initial attempts have been made in PD (Zhang et al., 2019) where three robust subtypes of PD were identified.

In summary, we created a deep learning architecture based on parameter-efficient building blocks like e.g. separable convolutions (which result in a 20-fold decrease in parameter utilization in a single mid-flow block) which is able to compress multiple voxel-wise brain images with minimal loss of information, excellent generalization abilities (in cross validation) and whose latent embeddings appear to map to meaningful and interesting phenotypic profiles. In view of possible clinical applications, the predictive validity of our clusters would need to be corroborated by longitudinal follow-ups in order to test whether the 'extreme' groups of people identified go on and develop clinically relevant disorders. If this is the case, this would strongly reinforce the idea that deep learning information compression based exclusively on neuroimaging has the ability to predict clinically relevant behavioural trajectories. We expect that our model will be able to aid in the current quest for solid avenues towards personalized medicine, i.e. the goal of creating an individual patient profile which is matched exactly as possible to a diagnosis, intervention and prognosis. Detecting generalizable patient strata from brain images only can aid in creating multi-dimensional biomarkers able to chart spatio-temporal trajectories of (possibly) identifiable pathophysiological mechanisms. It is hoped that such patients (sub)strata, identified through fully data-driven approaches, will correspond to e.g. different genetic makeups, different phenotypic groups, end eventually will respond differently to specific treatment strategies. If this were true, such results may be of aid in predicting disease evolution as well as drug response, hence also empowering clinical trials. In turn, this would provide stepping stones for the development of e.g. pharmacological treatments tailored to biomarker-guided homogeneous subgroups (or clusters).

\section{Acknowledgements}

Simeon Spasov research is supported by EPSRC. Luca Passamonti is funded by the Medical Research Council (MRC) grant (MR/P01271X/1) at the University of Cambridge, UK.

The GPUs on which this work was performed were generously provided by NVIDIA. 


\section{References}

Adler, C.H., Caviness, J.N., Sabbagh, M.N., Shill, H.A., Connor, D.J., Sue, L., Evidente, V.G., DriverDunckley, E. and Beach, T.G., 2010. Heterogeneous neuropathological findings in Parkinson's disease with mild cognitive impairment. Acta neuropathologica, 120(6), p.827.

Alashwal, Hany, et al. "The Application of Unsupervised Clustering Methods to Alzheimer's Disease." Frontiers in computational neuroscience 13 (2019).

Benjamini, Yoav, and Yosef Hochberg. "Controlling the false discovery rate: a practical and powerful approach to multiple testing." Journal of the Royal statistical society: series B (Methodological) 57.1 (1995): 289-300.

Brendan J. Frey et al., "Clustering by Passing Messages Between Data Points", Science Feb. 2007 Chollet, F. Xception: Deep learning with depthwise separable convolutions. In: Proceedings of the IEEE conference on computer vision and pattern recognition. 2017. p. 1251-1258.

Caruyer, E., Lenglet, C., Sapiro, G. and Deriche, R., 2013. Design of multishell sampling schemes with uniform coverage in diffusion MRI. Magnetic resonance in medicine, 69(6), pp.1534-1540

Cole, J. H., Poudel, R. P. K., Tsagkrasoulis, D., Caan, M. W. A., Steves, C., Spector, T. D., \& Montana, G. (2017a). Predicting brain age with deep learning from raw imaging data results in a reliable and heritable biomarker. NeuroImage, 163, 115-124. https://doi.org/10. 1016/j.neuroimage.2017.07.059:

Djork-Arné Clevert, Thomas Unterthiner, Sepp Hochreiter, Fast and Accurate Deep Network Learning by Exponential Linear Units (ELUs). 2015, CoRR abs/1511.07289

Glasser, M.F., Sotiropoulos, S.N., Wilson, J.A., Coalson, T.S., Fischl, B., Andersson, J.L., Xu, J., Jbabdi, S., Webster, M., Polimeni, J.R. and Van Essen, D.C., 2013. The minimal preprocessing pipelines for the Human Connectome Project. Neuroimage, 80, pp.105-124

Guan, Renchu, et al. "Text clustering with seeds affinity propagation." IEEE Transactions on Knowledge and Data Engineering 23.4 (2010): 627-637.

Havaei, Mohammad, et al. "Brain tumor segmentation with deep neural networks." Medical image analysis 35 (2017): 18-31.

He, K., Zhang, X., Ren, S., \& Sun, J. (2016). Deep residual learning for image recognition.

In Proceedings of the IEEE conference on computer vision and pattern recognition (pp. 770-778).

Howard, A G., et al. Mobilenets: Efficient convolutional neural networks for mobile vision applications. arXiv preprint arXiv:1704.04861, 2017.

Jack, C.R., Bennett, D.A., Blennow, K., Carrillo, M.C., Feldman, H.H., Frisoni, G.B., Hampel, H., Jagust, W.J., Johnson, K.A., Knopman, D.S. and Petersen, R.C., 2016. A/T/N: an unbiased descriptive classification scheme for Alzheimer disease biomarkers. Neurology, 87(5), pp.539-547.

Jennings, M.K. et al. (2011). The parkinson progression marker initiative (ppmi). Progress in neurobiology, 95(4):629--635.

Jenkinson, M., Beckmann, C.F., Behrens, T.E., Woolrich, M.W. and Smith, S.M., 2012. Fsl. Neuroimage, 62(2), pp.782-790.

Kolařík, Martin, et al. "Optimized high resolution 3d dense-u-net network for brain and spine segmentation." Applied Sciences 9.3 (2019): 404

S. Ioffe and C. Szegedy, 2015. Batch Normalization: Accelerating Deep Network Training by Reducing Internal Covariate Shift. 2015 International Conference on Machine Learning (ICML), p. 448-456

$\mathrm{Kao}, \mathrm{Po}-\mathrm{Yu}$, et al. "Improving Patch-Based Convolutional Neural Networks for MRI Brain Tumor Segmentation by Leveraging Location Information." Frontiers in Neuroscience 13 (2020): 1449.

Kingma, D.P. and Ba, J., 2014. Adam: A method for stochastic optimization. arXiv preprint arXiv: 1412.6980 .

Lasko, Thomas A., Joshua C. Denny, and Mia A. Levy. "Computational phenotype discovery using unsupervised feature learning over noisy, sparse, and irregular clinical data." PloS one 8.6 (2013).

LeCun, Yann, Yoshua Bengio, and Geoffrey Hinton. "Deep learning." nature 521.7553 (2015): 436444

Llera, Alberto, et al. "Inter-individual differences in human brain structure and morphology link to variation in demographics and behavior." Elife 8 (2019).

Lopez, Christian, et al. "An unsupervised machine learning method for discovering patient clusters based on genetic signatures." Journal of biomedical informatics 85 (2018): 30-39. 
bioRxiv preprint doi: https://doi.org/10.1101/2021.03.08.434427; this version posted March 13,2021 . The copyright holder for this preprint (which was not certified by peer review) is the author/funder. All rights reserved. No reuse allowed without permission.

Lundervold, Alexander Selvikvåg, and Arvid Lundervold. "An overview of deep learning in medical imaging focusing on MRI." Zeitschrift für Medizinische Physik 29.2 (2019): 102-127.

Minaee, Shervin, et al. "Image segmentation using deep learning: A survey." arXiv preprint arXiv:2001.05566 (2020).

Mwangi, Benson, Tian Siva Tian, and Jair C. Soares. "A review of feature reduction techniques in neuroimaging." Neuroinformatics 12.2 (2014): 229-244.

Mueller, S. G., et al. (2005). Ways toward an early diagnosis in Alzheimer's disease: the Alzheimer's disease neuroimaging initiative (ADNI). Alzheimer's \& Dementia, 1(1):55--66.

Rabinovici, G.D., Carrillo, M.C., Forman, M., DeSanti, S., Miller, D.S., Kozauer, N., Petersen, R.C., Randolph, C., Knopman, D.S., Smith, E.E. and Isaac, M., 2017. Multiple comorbid neuropathologies in the setting of Alzheimer's disease neuropathology and implications for drug development. Alzheimer's \& Dementia: Translational Research \& Clinical Interventions, 3(1), pp.8391.

Ronneberger, Olaf, Philipp Fischer, and Thomas Brox. "U-net: Convolutional networks for biomedical image segmentation." International Conference on Medical image computing and computer-assisted intervention. Springer, Cham, 2015.

Rousseeuw, Peter J. "Silhouettes: a graphical aid to the interpretation and validation of cluster analysis." Journal of computational and applied mathematics 20 (1987): 53-65.

Spasov, Simeon, et al. "A parameter-efficient deep learning approach to predict conversion from mild cognitive impairment to Alzheimer's disease." Neuroimage 189 (2019): 276-287.

Saha, Susmita, et al. "Predicting motor outcome in preterm infants from very early brain diffusion MRI using a deep learning convolutional neural network (CNN) model." NeuroImage (2020): 116807.

Nitish Srivastava, Geoffrey Hinton, Alex Krizhevsky, Ilya Sutskever, Ruslan Salakhutdinov, Yoshua Bengio, Dropout: A Simple Way to Prevent Neural Networks from Overfitting, Journal of Machine Learning Research (JMLR), p. 1929-1958 (2014)

Sotiropoulos, S.N., Jbabdi, S., Xu, J., Andersson, J.L., Moeller, S., Auerbach, E.J., Glasser, M.F., Hernandez, M., Sapiro, G., Jenkinson, M. and Feinberg, D.A., 2013. Advances in diffusion MRI acquisition and processing in the Human Connectome Project. Neuroimage, 80, pp.125-143

Sudlow, C., et al. (2015). Uk biobank: an open access resource for identifying the causes of a wide range of complex diseases of middle and old age. PLoS medicine,12(3):e1001779.

Taylor, J. R., et al. (2017). The Cambridge centre for ageing and neuroscience (CAM-can) data repository: structural and functional mri, meg, and cognitive data from a cross-sectional adult lifespan sample. Neuroimage, 144:262--269.

Toschi, N., Lista, S., Baldacci, F., Cavedo, E., Zetterberg, H., Blennow, K., Kilimann, I., Teipel, S.J., dos Santos, A.M., Epelbaum, S. and Lamari, F., 2019. Biomarker-guided clustering of Alzheimer's disease clinical syndromes. Neurobiology of aging, 83, pp.42-53.

Van Essen, D.C., Smith, S.M., Barch, D.M., Behrens, T.E., Yacoub, E., Ugurbil, K. and Wu-Minn HCP Consortium, 2013. The WU-Minn human connectome project: an overview. Neuroimage, 80, pp.62-79 Vlasblom, James, and Shoshana J. Wodak. "Markov clustering versus affinity propagation for the partitioning of protein interaction graphs." BMC bioinformatics 10.1 (2009): 99.

Yang, Qianye, et al. "MRI cross-Modality image-to-image translation." Scientific Reports 10.1 (2020): $1-18$.

Young, Jonathan D., Chunhui Cai, and Xinghua Lu. "Unsupervised deep learning reveals prognostically relevant subtypes of glioblastoma." BMC bioinformatics 18.11 (2017): 5-17.

Wang, Xin, et al. "Dissecting cancer heterogeneity-an unsupervised classification approach." The international journal of biochemistry \& cell biology 45.11 (2013): 2574-2579.

Wayne W., D. "Kruskal-Wallis one-way analysis of variance by ranks." Applied nonparametric statistics (1990): 226-234.

$\mathrm{Wu}$, Jia, et al. "Unsupervised clustering of quantitative image phenotypes reveals breast cancer subtypes with distinct prognoses and molecular pathways." Clinical Cancer Research 23.13 (2017): 3334-3342. Zhang, H., Schneider, T., Wheeler-Kingshott, C.A. and Alexander, D.C., 2012. NODDI: practical in vivo neurite orientation dispersion and density imaging of the human brain. Neuroimage, 61(4), pp.1000-1016. 
Zhang, X., Chou, J., Liang, J., Xiao, C., Zhao, Y., Sarva, H., Henchcliffe, C. and Wang, F., 2019. Datadriven subtyping of Parkinson's disease using longitudinal clinical records: a cohort study. Scientific reports, 9(1), pp.1-12.

Zoph, B., et al. Learning transferable architectures for scalable image recognition. In: Proceedings of the IEEE conference on computer vision and pattern recognition. 2018. p. 8697-8710. 